\title{
FDTD Modeling of a Novel ELF Radar for Major Oil Deposits Using a Three-Dimensional Geodesic Grid of the Earth-Ionosphere Waveguide
}

\author{
Jamesina J. Simpson, Student Member, IEEE, Ross P. Heikes, and Allen Taflove, Fellow, IEEE
}

\begin{abstract}
This paper reports the first application of an optimized geodesic, three-dimensional (3-D) finite-difference time-domain (FDTD) grid to model impulsive, extremely low-frequency (ELF) electromagnetic wave propagation within the entire Earth-ionosphere cavity. This new model, which complements our previously reported efficient 3-D latitude-longitude grid, is comprised entirely of hexagonal cells except for a small, fixed number of pentagonal cells. Grid-cell areas and locations are optimized to yield a smoothly varying area difference between adjacent cells, thereby maximizing numerical convergence. Extending from $100 \mathrm{~km}$ below sea level to an altitude of $100 \mathrm{~km}$, this technique can accommodate arbitrary horizontal as well as vertical geometrical and electrical inhomogeneities/anisotropies of the excitation, ionosphere, lithosphere, and oceans. We first verify the global model by comparing the FDTD-calculated daytime ELF propagation attenuation with data reported in the literature. Then as one example application of this grid, we illustrate a novel ELF radar for major oil deposits.
\end{abstract}

Index Terms-Earth, extremely low-frequency (ELF), finite-difference time-domain (FDTD), geodesic grid, oil field, propagation attenuation, radar, sphere, ultra-low frequency (ULF).

\section{INTRODUCTION}

$\mathbf{I}$ $\mathrm{N}$ this paper we discuss an advance in the emerging application of the finite-difference time-domain (FDTD) method for computational electrodynamics [1] to modeling transient ultra-low frequency (ULF: 0-3 Hz) and extremely-low frequency (ELF: $3 \mathrm{~Hz}-300 \mathrm{~Hz}$ ) electromagnetic wave propagation within the global Earth-ionosphere cavity. This technique permits a direct, three-dimensional (3-D), time-domain calculation of round-the-world ULF/ELF propagation accounting for arbitrary horizontal as well as vertical geometrical and electrical inhomogeneities and anisotropies of the excitation, ionosphere, lithosphere, and oceans. Specifically, we report here the use of a new optimized 3-D geodesic grid [1]-[3] having many advantages over our previously reported latitude-longitude grid model [4]-[9] for the study of ULF/ELF propagation. Possible applications of this grid are numerous, including the study of electromagnetic precursors to major earthquakes [7] and remote-sensing of major oil deposits [8], [9].

Manuscript received June 10, 2005; revised January 10, 2006. This work was supported in part by the National Computational Science Alliance under Grant DMS040006N and utilized the Dell Xeon Linux Cluster.

J. J. Simpson and A. Taflove are with the Electrical Engineering and Computer Science Department, McCormick School of Engineering, Northwestern University, Evanston, IL 60208 USA (e-mail: j-simpson@ northwestern.edu).

R. P. Heikes is with the Department of Atmospheric Science, Colorado State University, Fort Collins, CO 80521 USA.

Digital Object Identifier 10.1109/TAP.2006.875504
We previously reported applying FDTD to model the complete Earth-sphere at ELF using an efficient, 3-D, spherical-coordinate, latitude-longitude space lattice with periodic boundary conditions [4]-[9]. To minimize the effects of increasing space-cell eccentricity upon approaching a pole, we employed an adaptive cell-combination technique applied to adjacent grid cells in the east-west direction, thereby allowing maintenance of the time-step at nearly the level permitted by the Courant stability condition for the square equatorial cells. This technique permitted a standard laboratory computer with 2 GB of random access memory to generate high-resolution $(\sim 40 \times 40 \times 5 \mathrm{~km})$ modeling results for global, fully three-dimensional, impulsive ULF/ELF propagation within the entire Earth-ionosphere cavity to within $\pm 100 \mathrm{~km}$ of sea level. Comparing this grid's calculated daytime ELF propagation attenuation with the results of [10] showed agreement in the frequency range of $50-500 \mathrm{~Hz}$ to within about $\sim 0.5 \mathrm{~dB} / \mathrm{Mm}$ [5].

Here we report the first 3-D FDTD results for an alternative means to grid the Earth based upon a "geodesic" lattice arrangement. Such grids have been employed since the 1960s for the study of geophysical phenomena [11]. In particular, we construct an analogous grid to that currently being used for climate modeling [12]. A direct extension of our previously reported 2-D geodesic model of the Earth [3], this 3-D spherical FDTD grid is best described as resembling the surface of a soccer ball. It is comprised entirely of hexagonal cells except for a small fixed number of pentagonal cells [12]. Grid-cell areas and locations are optimized to yield a smoothly varying area difference between adjacent cells, thereby maximizing numerical convergence [13]. This model is superior to our previous latitude-longitude grid because it avoids geometrical singularities, executes 14-times faster with only a $40 \%$ increase in the required memory, and provides even more isotropic numerical wave propagation [3].

To verify this new model, we perform an analogous study to that in [5]. That is, we validate our 3-D geodesic FDTD model of the entire Earth-ionosphere cavity extending from 100 $\mathrm{km}$ below sea level to an altitude of $100 \mathrm{~km}$ by comparing the calculated daytime ELF propagation attenuation with that reported in [10]. Then as a first application of this grid, we provide numerical simulations of ELF radar returns from a hypothetical Alaskan oilfield excited by a $20-\mathrm{Hz}$ pulse emitted from the former U.S. Navy's Wisconsin Transmitting Facility (WTF). Our results indicate the possibility of detecting oil fields located within several $\mathrm{km}$ of the Earth's surface by recording only the 
TABLE I

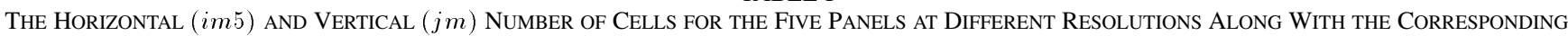
Distances BETwEEN ADJACENT GRID POINTS [14]

\begin{tabular}{|c|c|c|c|}
\hline $\begin{array}{c}\text { Total Number } \\
\text { of Cells }\end{array}$ & $\boldsymbol{i m 5}$ & jm & $\begin{array}{c}\text { Distance between } \\
\text { grid points }(\mathbf{k m})\end{array}$ \\
\hline 10,242 & 66 & 34 & 250.1 \\
\hline 40,962 & 130 & 66 & 125.1 \\
\hline 163,842 & 258 & 130 & 62.55 \\
\hline 655,362 & 514 & 258 & 31.27 \\
\hline $2,621,442$ & 1026 & 514 & 15.64 \\
\hline $10,485,762$ & 2050 & 1026 & 7.819 \\
\hline
\end{tabular}

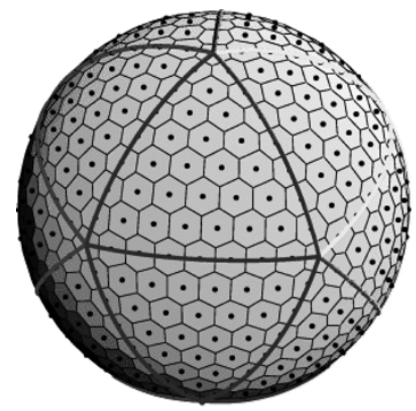

(a)

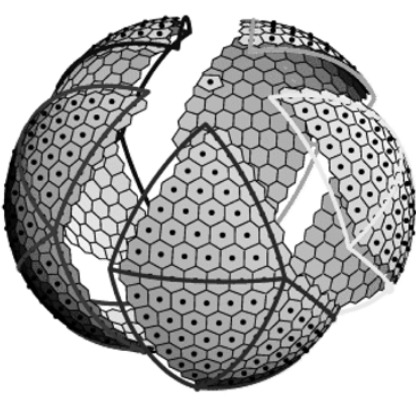

(b)

Fig. 1. General layout of a 642-cell, two-dimensional geodesic grid covering the complete Earth-sphere. Each grid cell is planar. The grid is divided into five equal panels with $i m 5=18$ and $j m=10$. (This figure previously appeared in [12] the Computing in Science and Engineering (CiSE) Magazine which is a joint publication of the IEEE Computer Society and the American Institute of Physics.)

radial (vertical) component of the scattered magnetic field $\left(H_{r}\right)$. We show that the $H_{r}$ component may provide a sensitive means to rapidly and inexpensively conduct aerial surveys of thousands of square $\mathrm{km}$ for significant oil deposits [8], [9].

\section{THE 3-D GEODESIC FDTD GRID}

Fig. 1 illustrates the distribution of cells for a sample geodesic grid, which for our purpose corresponds to the transverse-magnetic (TM) case of Maxwell's equations. This sample grid has a total of 642 planar cells, of which 630 are hexagons and 12 are pentagons. For purposes of efficient mapping into the computer memory, this grid can be divided into five equal panels of size $i m 5 \times j m$ cells, where $i m 5=18$ and $j m=10$, as described in [12]. Fig. 2(a) shows the five grid panels of Fig. 1 after unwrapping and stretching them flat. The cells at the North and South Poles can be arbitrarily connected to any of the five panels. The $(i=1, j=j m),(i=i m 5, j=j m)$, and $(i=i m 5, j=1)$ cells are dead cells, and the remainder of the cells in the $i=1$ and $i=i m 5$ rows and in the $j=1$ and $j=j m$ columns of each of the five panels correspond to ghost cells (see Section III-C). Fig. 2(b) then illustrates how the five grid panels of Fig. 1 can be assigned logically Cartesian coordinates, and further how all five panels can then be laid side-by-side to constitute an overall $(i m=90, j m=10)$ logically Cartesian grid. This powerful interpretation of a spherical geodesic grid for purposes of efficient computer processing can be implemented for a variety of meshing densities over the sphere, as shown in Table I [14].

Regardless of the grid resolution, we note that all but 12 of the cells for the TM case are planar hexagons. Although shown

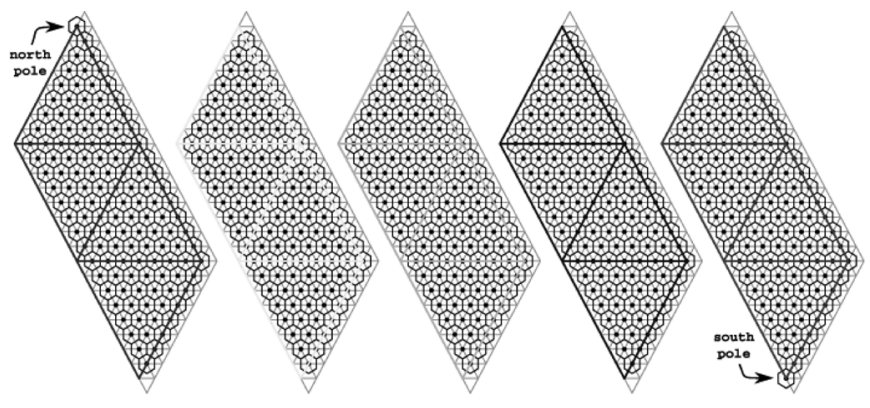

(a)
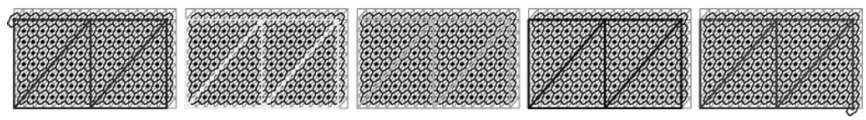

(b)

Fig. 2. (a) Five ( $i m 5=18, j m=10$ ) grid panels of Fig. 1 after unwrapping and stretching them flat. The grid cells at the North Pole and South Pole can be arbitrarily connected to any of the five panels. The $(i=1, j=j m),(i=i m 5$, $j=j m)$, and $(i=i m 5, j=1)$ cells are dead cells, and the remainder of the cells in the $i=1$ and $i=i m 5$ rows and in the $j=1$ and $j=j m$ columns of each of the five panels correspond to ghost cells (see Section III-C). Although shown as hexagons, the 12 pentagon cells are located at the North and South Poles, and at $(i=i m 5 / 2, j=j m-1)$ and $(i=i m 5-1, j=j m-1)$ on each of the five grid panels. (b) Illustration of how the cells in the five grid panels of Fig. 1 can be assigned logically Cartesian coordinates, and how all five panels can then be laid side-by-side to constitute an overall $(\mathrm{im}=90, \mathrm{jm}=10)$ logically Cartesian grid. (This figure previously appeared in [12] the Computing in Science and Engineering (CiSE) Magazine which is a joint publication of the IEEE Computer Society and the American Institute of Physics.)

as hexagons in Fig. 2, these 12 pentagon cells are located at the North and South Poles, and at $(i=i m 5 / 2, j=j m-1)$ and $(i=i m 5-1, j=j m-1)$ on each of the five grid panels. Each of the hexagonal cells has $\boldsymbol{E}$ and $\boldsymbol{H}$ components distributed as shown in Fig. 3(a). Fig. 3(b) then illustrates how the $\boldsymbol{E}$ and $\boldsymbol{H}$ components are distributed about each of the 12 planar pentagons.

A fully 3-D FDTD grid requires alternating planes of TM and transverse electric (TE) field components. Therefore, we define the TE plane as being comprised entirely of triangular cells centered at the vertices of each hexagonal and pentagonal cell of the TM plane. Fig. 4 illustrates how the $\boldsymbol{E}$ and $\boldsymbol{H}$ components are distributed about each of the triangular cells.

Reference [13] describes an optimization procedure for selecting the areas and locations of the TM hexagonal and pentagonal grid cells (and thereby also the TE triangular cells) on the sphere to ensure numerical consistency and to maximize the order of accuracy in the context of the Laplace operator. We have found that this optimization procedure also maximizes the 
$\odot E_{r}(i, j+1, k)$

(a)

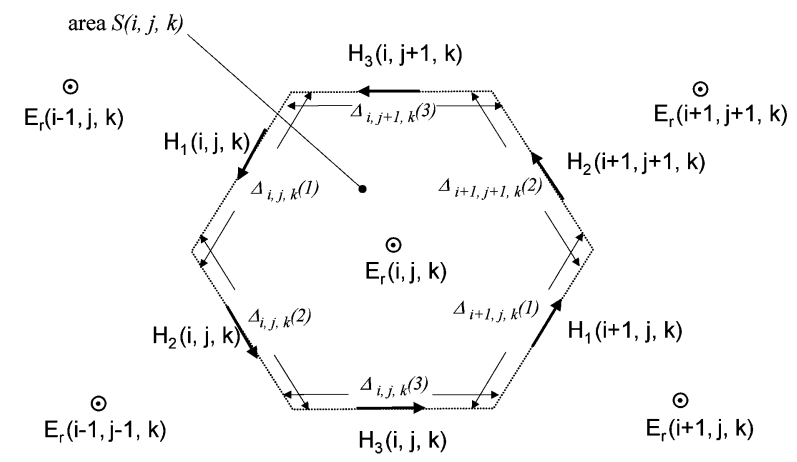

(b)

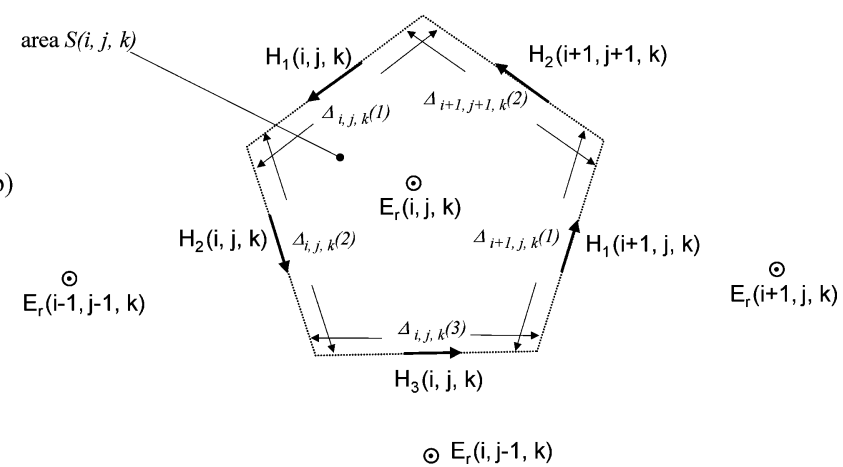

Fig. 3. Details of the grid-cell geometry for the $\mathrm{TM}_{\mathrm{r}}$ case: (a) hexagonal cell and (b) pentagonal cell.

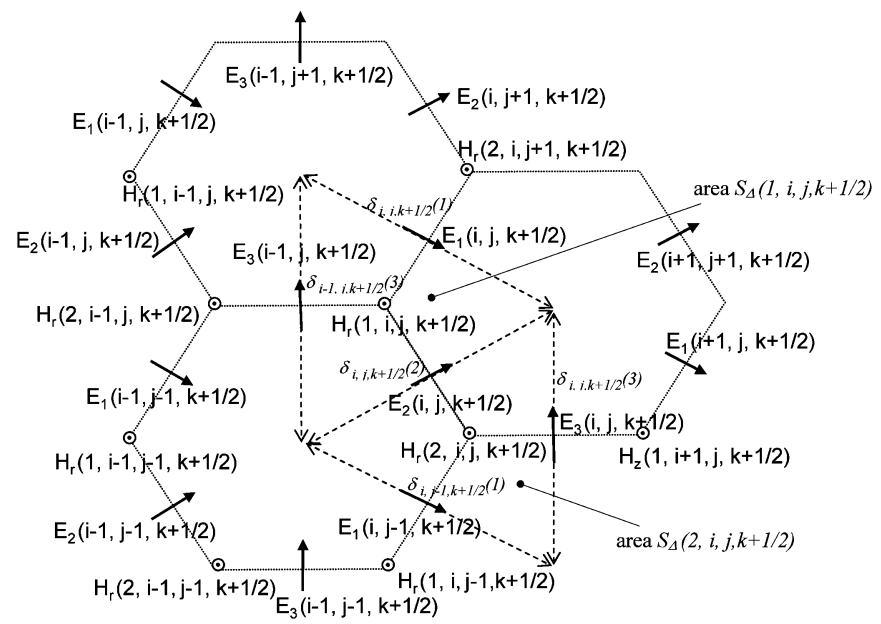

Fig. 4. Details of the $\mathrm{TE}_{\mathrm{r}}$ triangular grid cell geometry centered one-half cell above the vertices of the $\mathrm{TM}_{\mathrm{r}}$ hexagon/pentagon cells of Fig. 3 .

observed accuracy of wave propagation about the sphere as governed by Maxwell's equations [3].

In the following section we will discuss the updating equations for the fully 3-D geodesic FDTD grid, which is constructed by coupling the alternating TE and TM planes in the radial $(r)$-direction via regular Yee-type updates [1].

\section{THE FDTD ALGORITHM}

\section{A. Basic Algorithm, $\mathrm{TM}_{r}$ Components}

Ampere's Law in integral form [1] can be applied to develop an FDTD time-stepping relation for the electric field $E_{r}$ at the center of the $(i, j, k)$ th hexagonal grid cell. For example, referring to Fig. 3(a), for a hexagonal cell we have

$$
\begin{aligned}
E_{r}^{n+1}(i, j, k) \\
=E_{r}^{n}(i, j, k)+\frac{\Delta t}{\varepsilon_{0} S(i, j, k)} \\
\quad \times\left\{H_{1}^{n+0.5}(i, j, k) \Delta_{\mathrm{i}, \mathrm{j}, \mathrm{k}}(1)+H_{2}^{n+0.5}(i, j, k) \Delta_{\mathrm{i}, \mathrm{j}, \mathrm{k}}(2)\right. \\
\quad+H_{3}^{n+0.5}(i, j, k) \Delta_{\mathrm{i}, \mathrm{j}, \mathrm{k}}(3)-H_{1}^{n+0.5}(i+1, j, k) \\
\quad \times \Delta_{\mathrm{i}+1, \mathrm{j}, \mathrm{k}}(1)-H_{2}^{n+0.5}(i+1, j+1, k) \\
\left.\quad \times \Delta_{\mathrm{i}+1, \mathrm{j}+1, \mathrm{k}}(2)-H_{3}^{n+0.5}(i, j+1, k) \Delta_{\mathrm{i}, \mathrm{j}+1, \mathrm{k}}(3)\right\}
\end{aligned}
$$

where $\Delta t$ is the time-step, $\Delta_{i, j, k}(n)$ is the $n$th wall length of the cell centered around $E_{r}(i, j, k)$ (where $n$ is equal to one, two, or three), and $S(i, j, k)$ is the area of the cell centered around $E_{r}(i, j, k)$.

Similarly, referring to Fig. 3(b), the update for $E_{r}$ at the center of a pentagonal cell at mid-latitude is given by

$$
\begin{aligned}
& E_{r}^{n+1}(i, j, k) \\
& =E_{r}^{n}(i, j, k)+\frac{\Delta t}{\varepsilon_{0} S(i, j, k)} \\
& \quad \times\left\{H_{1}^{n+0.5}(i, j, k) \Delta_{\mathrm{i}, \mathrm{j}, k}(1)+H_{2}^{n+0.5}(i, j, k)\right. \\
& \quad \times \Delta_{\mathrm{i}, \mathrm{j}, k}(2)+H_{3}^{n+0.5}(i, j, k) \Delta_{\mathrm{i}, \mathrm{j}, k}(3) \\
& \quad-H_{1}^{n+0.5}(i+1, j, k) \Delta_{\mathrm{i}+1, \mathrm{j}, k}(1) \\
& \left.\quad-H_{2}^{n+0.5}(i+1, j+1, k) \Delta_{\mathrm{i}+1, \mathrm{j}+1, k}(2)\right\} .
\end{aligned}
$$

In the same manner, the updates for $E_{r}$ at the center of a pentagonal cell at the North and South Poles are given respectively by

$$
\begin{aligned}
& E_{r}^{n+1}(1, j m-1, k) \\
& =E_{r}^{n}(1, j m-1, k)+\frac{\Delta t}{\varepsilon_{0} S(1, j m-1, k)} \\
& \times\left\{-H_{1}^{n+0.5}(2, j m-1, k) \Delta_{2, \mathrm{jm}-1, k}(1)\right. \\
& -H_{1}^{n+0.5}(\mathrm{im} 5+2, j m-1, k) \Delta_{\mathrm{im} 5+2, \mathrm{jm}-1, k}(1) \\
& -H_{1}^{n+0.5}(i m 5 * 2+2, j m-1, k) \Delta_{\mathrm{im} 5 * 2+2, \mathrm{jm}-1, k}(1) \\
& -H_{1}^{n+0.5}(\mathrm{im} 5 * 3+2, j m-1, k) \Delta_{\mathrm{im} 5 * 3+2, \mathrm{jm}-1, k}(1) \\
& \left.-H_{1}^{n+0.5}(i m 5 * 4+2, j m-1, k) \Delta_{\mathrm{im} 5 * 4+2, \mathrm{jm}-1, k}(1)\right\}
\end{aligned}
$$

$$
\begin{aligned}
& E_{r}^{n+1}(i m-1,1, k) \\
& \begin{aligned}
=E_{r}^{n}(i m-1,1, k)+\frac{\Delta t}{\varepsilon_{0} S(i m-1,1, k)} \\
\quad \times\left\{-H_{3}^{n+0.5}(i m 5-1,2, k) \Delta_{\mathrm{im} 5-1,2, k}(3)\right. \\
\quad-H_{3}^{n+0.5}(i m 5 * 2-1,2, k) \Delta_{\mathrm{im} 5 * 2-1,2, k}(3) \\
\quad-H_{3}^{n+0.5}(i m 5 * 3-1,2, k) \Delta_{\mathrm{im} 5 * 3-1,2, k}(3) \\
\quad-H_{3}^{n+0.5}(i m 5 * 4-1,2, k) \Delta_{\mathrm{im} 5 * 4-1,2, k}(3) \\
\left.\quad-H_{3}^{n+0.5}(i m 5 * 5-1,2, k) \Delta_{\mathrm{im} 5 * 5-1,2, k}(3)\right\}
\end{aligned}
\end{aligned}
$$


where the $\Delta_{\mathrm{i}, \mathrm{j}, \mathrm{k}}(n)$ and the $H_{1}$ or $H_{3}$ component having the indicated coordinates from each of the five panels are used.

The basic FDTD time-stepping algorithm for the $\mathrm{TM}_{\mathrm{r}}$ components is completed by specifying the updates for the $H_{1}, H_{2}$, and $H_{3}$ fields using Faraday's Law in integral form [1]. For example, referring to the grid cells shown in Figs. 3 and 4, we have

$$
\begin{aligned}
H_{1}^{n+1} & (i, j, k) \\
= & H_{1}^{n+0.5}(i, j, k)+\frac{\Delta t}{\mu_{0} \delta_{i, j, k}(1)} \\
\times & {\left[E_{r}^{n+1}(i-1, j, k)-E_{r}^{n+1}(i, j, k)\right]+\frac{\Delta t}{\mu_{0} \Delta r} } \\
\times & {\left[E_{1}^{n+1}\left(i, j, k+\frac{1}{2}\right)-E_{1}^{n+1}\left(i, j, k-\frac{1}{2}\right)\right] } \\
H_{2}^{n+1.5}(i, j, k) & \\
= & H_{2}^{n+0.5}(i, j, k)+\frac{\Delta t}{\mu_{0} \delta_{\mathrm{i}, \mathrm{j}, k}(2)} \\
\times & {\left[E_{r}^{n+1}(i-1, j-1, k)-E_{r}^{n+1}(i, j, k)\right]+\frac{\Delta t}{\mu_{0} \Delta r} } \\
\times & {\left[E_{2}^{n+1}\left(i, j, k+\frac{1}{2}\right)-E_{2}^{n+1}\left(i, j, k-\frac{1}{2}\right)\right] } \\
H_{3}^{n+1.5} & (i, j, k) \\
= & H_{3}^{n+0.5}(i, j, k)+\frac{\Delta t}{\mu_{0} \delta_{\mathrm{i}, \mathrm{j}, k}(3)} \\
\times & {\left[E_{r}^{n+1}(i, j-1, k)-E_{r}^{n+1}(i, j, k)\right]+\frac{\Delta t}{\mu_{0} \Delta r} } \\
\times & {\left[E_{3}^{n+1}\left(i, j, k+\frac{1}{2}\right)-E_{3}^{n+1}\left(i, j, k-\frac{1}{2}\right)\right] }
\end{aligned}
$$

where $\Delta r$ is the lattice space cell increment in the radial direction and $\delta_{i, j, k}(n)$ is the $n$th distance between adjacent $E_{r}$ components as shown for one-half cell above the $\mathrm{TM}_{r}$ plane in Fig. 4.

\section{B. Basic Algorithm, $\mathrm{TE}_{r}$ Components}

Faraday's Law in integral form [1] can be applied to develop an FDTD time-stepping relation for the $H_{\mathrm{r}}$ field at the center of the $(i, j, k+(1 / 2))$ th triangular grid cell, one-half cell directly above the vertex $v$ of the $(i, j, k)$ th hexagonal/pentagonal grid cell (where $v$ is equal to one or two). For example, referring to Fig. 4, we have

$$
\begin{aligned}
& H_{r}^{n+1.5}\left(1, i, j, k+\frac{1}{2}\right) \\
& =H_{r}^{n+0.5}\left(1, i, j, k+\frac{1}{2}\right)+\frac{\Delta t}{\mu_{0} S_{\Delta}\left(1, i, j, k+\frac{1}{2}\right)} \\
& \quad \times\left\{E_{1}^{n+1}\left(i, j, k+\frac{1}{2}\right) \delta_{\mathrm{i}, \mathrm{j}, k+\frac{1}{2}}(1)\right. \\
& \quad-E_{2}^{n+1}\left(i, j, k+\frac{1}{2}\right) \delta_{\mathrm{i}, \mathrm{j}, k+\frac{1}{2}}(2) \\
& \left.\quad+E_{3}^{n+1}\left(i-1, j, k+\frac{1}{2}\right) \delta_{\mathrm{i}-1, \mathrm{j}, k+\frac{1}{2}}(3)\right\}
\end{aligned}
$$

$$
\begin{aligned}
& H_{r}^{n+1.5}\left(2, i, j, k+\frac{1}{2}\right) \\
&= H_{r}^{n+0.5}\left(2, i, j, k+\frac{1}{2}\right)+\frac{\Delta t}{\mu_{0} S_{\Delta}\left(2, i, j, k+\frac{1}{2}\right)} \\
& \quad \times\left\{E_{2}^{n+1}\left(i, j, k+\frac{1}{2}\right) \delta_{\mathrm{i}, \mathrm{j}, k+\frac{1}{2}}(2)\right. \\
& \quad-E_{3}^{n+1}\left(i, j, k+\frac{1}{2}\right) \delta_{\mathrm{i}, \mathrm{j}, k+\frac{1}{2}}(3) \\
&\left.\quad-E_{1}^{n+1}\left(i, j-1, k+\frac{1}{2}\right) \delta_{\mathrm{i}, \mathrm{j}-1, k+\frac{1}{2}}(1)\right\}
\end{aligned}
$$

where $\delta_{i, j, k+(1 / 2)}(n)$ is the $n$th wall length of the triangular cell centered around $H_{z}(v, i, j, k+(1 / 2))$ and $S_{\Delta}(v, i, j, k+(1 / 2))$ is the area of the triangular cell centered around $H_{z}(v, i, j, k+$ $(1 / 2))$.

The basic FDTD time-stepping algorithm for the $\mathrm{TE}_{\mathrm{r}}$ components is completed by specifying the electric field updates using Ampere's Law in integral form [1]. For example, referring to Fig. 4, we have

$$
\begin{aligned}
E_{1}^{n+1}\left(i, j, k+\frac{1}{2}\right)= & E_{1}^{n}\left(i, j, k+\frac{1}{2}\right)+\frac{\Delta t}{\varepsilon_{0} \Delta_{\mathrm{i}, \mathrm{j}, k+\frac{1}{2}}(1)} \\
& \times\left[H_{r}^{n+0.5}\left(2, i, j+1, k+\frac{1}{2}\right)\right. \\
& \left.-H_{r}^{n+0.5}\left(1, i, j, k+\frac{1}{2}\right)\right] \\
& +\frac{\Delta t}{\varepsilon_{0} \Delta r}\left[H_{1}^{n+0.5}(i, j, k+1)\right. \\
E_{2}^{n+1}\left(i, j, k+\frac{1}{2}\right)= & E_{2}^{n}\left(i, j, k+\frac{1}{2}\right)+\frac{\Delta t}{\varepsilon_{0} \Delta_{\mathrm{i}, \mathrm{j}, k+\frac{1}{2}}(2)} \\
& \times\left[H_{r}^{n+0.5}\left(1, i, j, k+\frac{1}{2}\right)\right. \\
& \left.-H_{r}^{n+0.5}(i, j, k)\right] \\
+ & \frac{\Delta t}{\varepsilon_{0} \Delta r}\left[H_{2}^{n+0.5}(i, j, k+1)\right. \\
& \left.\quad-H_{2}^{n+0.5}(i, j, k)\right] \\
E_{3}^{n+1}\left(i, j, k+\frac{1}{2}\right)= & E_{3}^{n}\left(i, j, k+\frac{1}{2}\right)+\frac{\Delta t}{\varepsilon_{0} \Delta_{\mathrm{i}, \mathrm{j}, k+\frac{1}{2}}(3)} \\
& \times\left[H_{r}^{n+0.5}\left(2, i, j, k+\frac{1}{2}\right)\right. \\
& \left.\quad-H_{r}^{n+0.5}\left(1, i+1, j, k+\frac{1}{2}\right)\right] \\
\varepsilon_{0} \Delta r & \left.-H_{3}^{n+0.5}(i, j, k)\right]
\end{aligned}
$$

where $\Delta_{i, j, k+}(1 / 2)(n)$ is the $n$th distance between adjacent $H_{\mathrm{r}}$ components as shown for $1 / 2$ cell below the $\mathrm{TE}_{\mathrm{r}}$ plane in Fig. 3 .

\section{Grid Wrap-Around (Periodic Boundary Condition)}

All field components on each of the five panels for which $2<i<(i m 5-1)$ and $2<j<(j m-1)$, along with the 
components at the centers of the pentagons at the North and South Poles are updated according to the algorithm presented in above. The ghost $E$ and $H$ field components, i.e., for $(i=1$, $1<j<j m-2),(1<i<i m 5-2, j=1),(i=i m 5$, $2<j<j m-1)$, and $(2<i<i m 5-1, j=j m)$ on all five panels, as well as for $(i=1, j=j m-1)$ and $(i=i m 5-1$, $j=1$ ) on the four panels not updating the North and South Poles, are filled after each time step by setting them equal to the corresponding component of the neighboring panel.

\section{VALIDATION STUDY}

In this section, our goal is to validate the new 3-D geodesic FDTD model by comparing its predicted ELF propagation characteristics with the data reported in [10]. Our model utilizes topographic and bathymetric data from the NOAA-NGDC "Global Relief CD-ROM" [15]. These data are mapped onto our 3-D space lattice having 163,842 total cells at each radial coordinate, yielding an assumed resolution of about 63 $\mathrm{km}$ between adjacent hexagonal/pentagonal cell centers (see Table I). In the radial direction, there are 40 grid cells, yielding a resolution of $5 \mathrm{~km}$ and a total of over 6.5 million cells for the entire 3-D grid.

For the lithosphere, conductivity values are assigned according to [16], depending upon the depth of the space lattice point and whether it is located directly below an ocean or within a continent. For the atmosphere, daytime exponential conductivity profiles of [17] are assumed. This permits the most straightforward comparison of the present FDTD modeling results with the data reported in [10] since ELF propagation is crucially affected by the ionosphere characteristics. Note that, however, the present model is capable of significantly greater ionospheric detail (i.e., day-to-night transitions, anisotropy, etc.) than that possible using the analysis of [10].

We excite our model with a vertical, 5-km-long current pulse having a Gaussian time-waveform with a 1/e full-width of 480 $\Delta t$, where $\Delta t=3.0 \mu \mathrm{s}$. To ensure a smooth onset of the excitation, the temporal center of this pulse is at $960 \Delta t$. This current pulse is located just above the Earth's surface on the equator at longitude $47^{\circ} \mathrm{W}$ (off the coast of South America).

Fig. 5 graphs time-waveforms of the FDTD-calculated radial E-field at four observation points: $A$ and $A^{\prime}$ on the Earth's surface directly east and west of the source at 1/4 of the distance to the antipode along the equator, respectively; and points $B$ and $B^{\prime}$ directly east and west of the source at $1 / 2$ of the distance to the antipode along the equator, respectively. Although the east and west waveforms are similar in appearance for both cases $\left(A\right.$ and $A^{\prime} ; B$ and $B^{\prime}$ ), they are not identical. The observed lack of symmetry is caused by a corresponding lack of symmetry of the lithosphere geometry in this region. We also note that the differences between the four waveforms reported here and the corresponding waveforms obtained previously with our latitude-longitude grid are due to the lower resolution of the geodesic grid (65 km versus $40 \mathrm{~km}$ between adjacent grid points in the east-west direction) [5] and therefore requiring different topographical data values, as well as the higher-degree of isotropic wave propagation achievable with the geodesic grid [3]. Finally, in the waveforms of Fig. 5, we note the evolution of a "slow-tail" response similar to that predicted in [18].

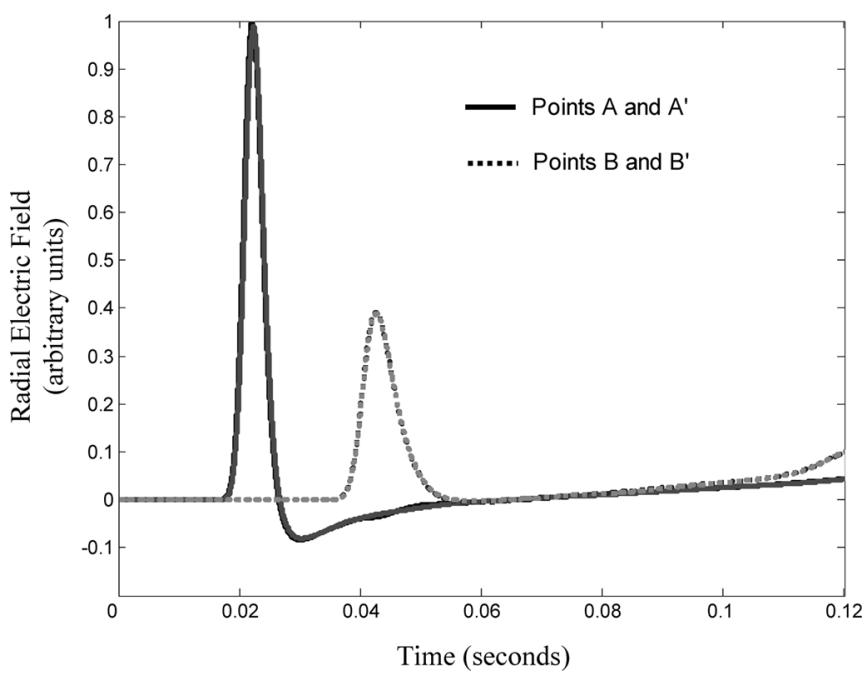

Fig. 5. FDTD-calculated temporal response observed at the equator directly east and west of the source for daytime ionosphere conductivity: (a) at points $A$ and $A^{\prime}$ located $1 / 4$ of the distance to the antipode and (b) at points $B$ and $B^{\prime}$ located at $1 / 2$ of the distance to the antipode.

Fig. 6 compares the daytime FDTD-calculated ELF propagation attenuation versus frequency over paths $A B$ and $A^{\prime} B^{\prime}$ with the theoretical results reported in [10], which were shown to have excellent agreement with experimentally determined values of ELF daytime attenuation rates. The FDTD data are obtained by forming the ratio of the discrete Fourier transforms (DFTs) of the time-waveforms shown in Fig. 5, with each of the waveforms truncated near 0.08 s (i.e., at each waveform's zerocrossing). ${ }^{1}$ Over the frequency range $50-500 \mathrm{~Hz}$, the FDTDcomputed propagation attenuation values agree with the results of [10] to within about $\pm 0.5 \mathrm{~dB} / \mathrm{Mm}$ over both paths $A B$ and $A^{\prime} B^{\prime}$.

\section{ELF RADAR FOR MAJOR OIL DEPOSITS}

We now illustrate one of many applications for the 3-D FDTD geodesic grid described above: a novel ELF radar for major oil deposits. Over many decades, several methods have been developed for performing subsurface conductivity measurements to locate and characterize natural resources (see for example the extensive survey in [19]). Only very few of the existing methods, however, can probe very deep into the Earth's crust, and fewer still employ controlled sources. In this paper, we are interested in techniques capable of exploring deep within the Earth's crust (up to $\sim 15 \mathrm{~km}$ ) using well-characterized controlled (man-made) sources, as opposed to natural sources having a random occurrence and random properties. Previously, controlled sources such as power lines, electrified railroads, and pulsed magnetohydrodynamic (MHD) generators have been used for such probing [20]. However, the data obtained from these methods exhibits an

\footnotetext{
${ }^{1}$ We must window the FDTD-calculated time-waveforms in this manner because the slow-tail response persists after the appearance of the signal arriving over the equatorial long path. Therefore, the slow-tail response cannot be timestepped to its conclusion in isolation. The required windowing process in time results in a frequency window of approximately $50-500 \mathrm{~Hz}$ within which our FDTD results can be validly compared with the benchmark data of [10]. This is because errors in the DFTs occur below about $50 \mathrm{~Hz}$ due to the absence of the slow-tail response, while errors occur above about $500 \mathrm{~Hz}$ due to the truncation of the data records.
} 


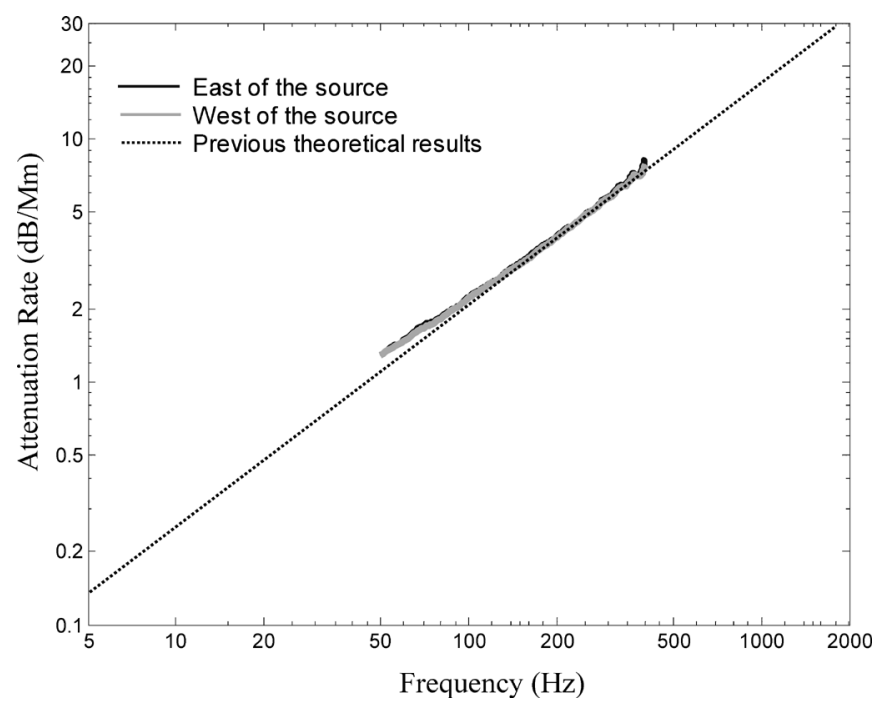

Fig. 6. Comparison between the FDTD-calculated daytime ELF propagation attenuation versus frequency between the 1/4 and 1/2-way points to the antipode both east and west of the source along the equator with the theoretical results reported in [10]. For both propagation paths, agreement to within about \pm 0.5 $\mathrm{dB} / \mathrm{Mm}$ is achieved over the frequency range of $50-500 \mathrm{~Hz}$.

unsatisfactory, wide variance [20]. Further, the above techniques can only be applied at considerable cost and effort, making their implementation infeasible in many regions of the world. As a result, new approaches to deep EM geophysical prospecting are desirable. If a superior technique applicable on a global scale were developed, it could permit both rapid and inexpensive surveying of the entire Earth's crust. Such a method would be of particular interest for locating anomalous conductivity structures such as mineral deposits and major oilfields.

Since the early 1990s a promising new system for global remote sensing has been under investigation [20], [21]. This technique involves the use of a powerful radio-transmitting antenna in the ELF range. In [20], Velikhov et al. outlined the many advantages of employing ELF radio waves up to $100 \mathrm{~Hz}$ for EM sounding.

1) Sufficiently large skin depth for deep probing (100 m in ocean and 10-15 km in crystalline shields).

2) Low propagation attenuation, which could provide global remote sensing of the Earth with a single source.

3) Good stability compared to higher frequency methods that are more influenced by ionospheric disturbances.

Due to these particular properties of ELF waves, Velikhov et al. introduced the idea of simultaneously performing studies around the globe involving oil-bearing rock, seismic regions, ionospheric wave propagation, and other areas of geophysics using one powerful ELF source.

The ELF sounding methodology described in [20], [21] however, involves electromagnetic field impedance measurements to determine the resistivity of the underlying rock. This requires measurement of both the tangential electric field, $\mathrm{E}_{\mathrm{tan}}$, and the tangential magnetic field, $H_{\mathrm{tan}}$, near the Earth's surface. In the following section, we describe a novel ELF radar for major oil deposits that requires detection of only the radial magnetic field, $H_{\mathrm{r}}$, as a low-frequency pulse is radiated outward from a distant, powerful ELF antenna [8], [9].

\section{ELF RADAR MODELING DETAILS AND RESUlTS}

Our goal is to determine how the presence of a major oil field influences the surface $H$-field components when excited by an ELF pulse radiated from a distant transmitter. For this study, we model an oil field as a lithosphere conductivity anomaly having lateral area of $4800 \mathrm{~km}^{2}$ and thickness $1.25 \mathrm{~km}$ located in Alaska at $156^{\circ} \mathrm{W}, 69^{\circ} \mathrm{N}$ (subgridding [1] is used to refine the radial grid resolution in the lithosphere near the Earth's surface to $1.25 \mathrm{~km}$, a factor of 4 times finer than the nominal radial resolution of $5 \mathrm{~km}$ used in the atmosphere and deeper within the lithosphere). The anomaly is assumed to have a conductivity that is ten times lower than that of the surrounding strata. By way of comparison, Alaska's Kuparuk River oil field spans a lateral area of about $1000 \mathrm{~km}^{2}$ [22], but this radar technique has been shown to be successful for detecting conductivity anomalies of that size [8], [9].

For the distant ELF transmitter, we employ the ELF WTF, a section of the former Navy's ELF communication system (in operation until September 2004 [23]) near Clam Lake, WI $\left(90.9^{\circ} \mathrm{W}, 46.5^{\circ} \mathrm{N}\right)$ for detecting the lithosphere conductivity anomaly in Alaska (at a distance of about 4.4 Mm). We assume nominal operation of this facility with two orthogonal ground lines, each $22.5 \mathrm{~km}$ long and carrying a current of $300 \mathrm{~A}$, one oriented in the north-south direction and the other in the East-West direction. However, our assumed carrier frequency is $20 \mathrm{~Hz}$, rather than the $75-\mathrm{Hz}$ Navy frequency. We further assume that the transmitted signal is a pulse that is generated by double-sideband amplitude modulation of the $20-\mathrm{Hz}$ carrier by a Gaussian envelope waveform of full-width at half-maximum 42.5 msec. Below the WTF, we model the Laurentian Plateau (or Canadian Shield), a large region of low-conductivity rock of 2.4E-4 S/m that extends northward from the Great Lakes toward the Arctic Ocean and includes much of Canada and Greenland.

For this study, we use two separate FDTD models to calculate the time-waveforms of the $H_{\mathrm{tan}}$ and $H_{r}$ components at a point $P$ at the Earth's surface directly above the conductivity anomaly. Model A serves to provide the reference time-waveforms (i.e., no conductivity anomaly present). Model B provides the time-waveforms for the case of the conductivity anomaly assumed to be located at a median depth of $1.2 \mathrm{~km}$. Both models employ the lithosphere and daytime ionosphere conductivity values described in Section IV. In addition, both models include conductivity values for the Laurentian Plateau mapped into the North American continental model. We define the normalized field-perturbation waveform $\Delta H(\mathrm{t})$ due to the conductivity anomaly as the absolute value of the difference between the time-waveforms of Models A and B divided by the peak value of the Model-A waveform.

Using a decibel scale, Fig. 7 shows our calculated results for $\Delta H(\mathrm{t})$ at surface observation point $\mathrm{P}$ due only to the $4800 \mathrm{~km}^{2}$ conductivity anomaly at a median depth of $1.2 \mathrm{~km}$. The following two cases are illustrated: a) $\Delta H_{\tan }(\mathrm{t})$ and b) $\Delta H_{r}(\mathrm{t})$. We see that $\Delta H_{\mathrm{tan}}(\mathrm{t})$ is weaker than the reference $H_{\mathrm{tan}}$ by more than $25 \mathrm{~dB}$ at almost every time point. On the other hand, $\Delta H_{r}(\mathrm{t})$ can be as much as $20 \mathrm{~dB}$ stronger than the reference $H_{r}$. Combined with our calculations that show that $\Delta H_{\tan }(\mathrm{t})$ 


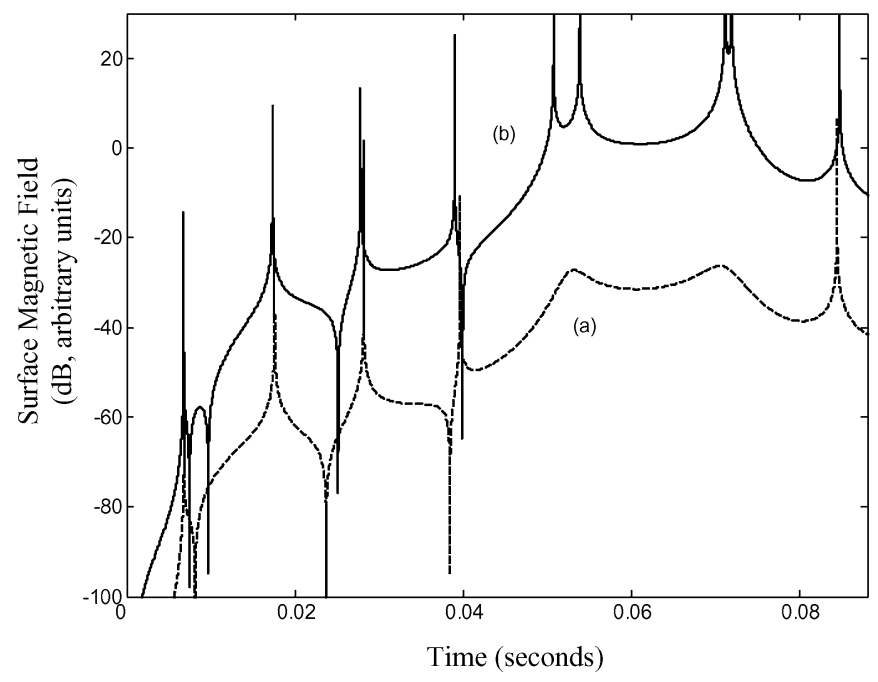

Fig. 7. Comparison of the FDTD-calculated results for the absolute values of the normalized field-perturbation waveform $\Delta H(\mathrm{t})$ at surface observation point $P$ due only to the $4800 \mathrm{~km}^{2}$ conductivity anomaly at a median depth of 1.2 $\mathrm{km}$. The following two cases are illustrated: (a) $\Delta H_{\tan }(\mathrm{t})$; (b) $\Delta H_{r}$ (t). The spikes are due to zero-crossings of the reference time-waveforms (i.e., without the conductivity present) used to normalize the field-perturbation waveforms.

and $\Delta H_{r}(\mathrm{t})$ are comparable in strength for the modeled conductivity anomaly, we conclude that measuring $H_{r}$ perturbations can yield an approximate $45 \mathrm{~dB}$ improvement in sensitivity relative to measuring $H_{\tan }$ perturbations. Therefore, since $H_{\text {tan }}$ is required for the impedance measurement technique reported in [20], [21], Fig. 7 indicates that the sensitivity of the impedance technique is considerably less than afforded by a measurement of the $H_{r}$ perturbation. Furthermore, we find from analogous studies involving deeper conductivity anomalies that measuring the $H_{r}$ perturbation becomes increasingly superior for such structures located at greater depths. Given the present results and those of [21], it appears possible to detect $H_{r}$ perturbations for conductivity anomalies located as deep as 10-20 $\mathrm{km}$.

One implication of the results shown in Fig. 7 is that a powerful, distant ELF antenna can be used to locate oil-bearing rock within several $\mathrm{km}$ of the Earth's surface by measuring the highly sensitive surface $H_{\mathrm{r}}$. We therefore propose that an ELF source such as the WTF, used in conjunction with an airborne magnetometer having sufficient sensitivity to $H_{\mathrm{r}}$, can be used to rapidly and inexpensively detect oilfields over thousands of square $\mathrm{km}$ [8], [9]. In fact, considering the very low propagation attenuation of electromagnetic waves at ELF as shown in Fig. 6, employing a sufficiently powerful ELF antenna permits use of this sounding technique on a global scale.

We suggest the use of SQUID magnetometers for the aerial surveys described in this paper because they: 1) measure the magnetic field directly; 2) offer higher sensitivity at frequencies less than $150 \mathrm{~Hz}$ than other receiver technologies, and (3) provide higher bandwidths [24]. Further, they have already been successfully implemented in aerial surveys [24].

\section{CONCLUSION AND ONGOING WORK}

We have presented a new geodesic FDTD grid model for 3-D electromagnetic wave propagation about the Earth-sphere that is considerably superior to our previously reported latitude-longitude grid [4]-[9]. Both the geodesic and latitude-longitude grids can account for arbitrary horizontal as well as vertical geometrical and electrical inhomogeneities/anisotropies of the excitation, ionosphere, lithosphere, and oceans. The new geodesic grid model, however, is simpler to construct, avoids geometrical singularities at the poles, executes about 14-times faster, and provides much more isotropic wave propagation. It may also permit an easier interchange of data with state-of-the-art Earth-simulation codes widely used by the geophysics community [3].

We first verified the new geodesic grid reported here by comparing the daytime FDTD-calculated round-the-world ELF propagation attenuation with data reported in the literature [10]. Then as an initial application of our new 3-D geodesic model, we illustrated a novel ELF radar for major oil deposits [8], [9]. Numerical simulations of ELF radar returns from a hypothetical Alaskan oilfield excited by a $20-\mathrm{Hz}$ pulse emitted from the former U.S. Navy site in Wisconsin, illustrated that the surface $H_{\mathrm{r}}$ exhibits an unexpected and very high degree of sensitivity to the presence of deeply buried conductivity anomalies of the lithosphere, much more so than $H_{\mathrm{tan}}$. We therefore proposed that airborne SQUID magnetometers recording only $H_{\mathrm{r}}$, synchronized with the transmission of an ELF pulse from a distant, powerful ELF antenna, may potentially provide the global locations of oilfields in a rapid and inexpensive manner. In ongoing work, we are considering the impact of ambient electromagnetic noise (e.g., telluric currents, man-made noise, and natural magnetic flux) upon our proposed technique.

\section{REFERENCES}

[1] A. Taflove and S. C. Hagness, Computational Electrodynamics: The Finite-Difference Time- Domain Method, 3rd ed. Norwood, MA: Artech House, 2005.

[2] J. J. Simpson and A. Taflove, "3-D FDTD modeling of ULF/ELF propagation within the global Earth-ionosphere cavity using an optimized geodesic grid," in Proc. IEEE AP-S Int. Symp., Washington, D.C., Jul. 2005, vol. 3A, pp. 346-349.

[3] J. J. Simpson and A. Taflove, "Efficient modeling of impulsive ELF antipodal propagation about the Earth sphere using an optimized twodimensional geodesic FDTD grid," IEEE Antennas Wireless Propag. Lett., vol. 3, no. 11, pp. 215-218, 2004.

[4] J. Simpson and A. Taflove, "Two-dimensional FDTD model of antipodal ELF propagation and Schumann resonance of the Earth," IEEE Antennas Wireless Propag. Lett., vol. 1, pp. 53-56, 2002.

[5] J. J. Simpson and A. Taflove, "Three-dimensional FDTD modeling of impulsive ELF propagation about the Earth-sphere," IEEE Trans. Antennas Propag., vol. 52, pp. 443-451, Feb. 2004.

[6] World Wide Web URL [Online]. Available: http://www.ece.northwestern.edu/ecefaculty/taflove/3Dmovietext@gif.avi

[7] J. J. Simpson and A. Taflove, "Entire-earth FDTD modeling of electrokinetic effects prior to the Loma Prieta earthquake," Geophys. Res. Lett., vol. 32, p. L09302, doi:10.1029/2005GL022601.

[8] _ , "Whole earth FDTD modeling of ELF electromagnetic sounding of oil deposits with the U.S. Navy's ELF transmitter system," in USNC/ URSI Nat. Radio Science Meeting, Washington, D.C., Jul. 2005.

[9] —_, "A novel ELF radar for major oil deposits," IEEE Geosci. Remote Sensing Lett., vol. 3, no. 1, pp. 36-39, 2006.

[10] P. Bannister, "ELF propagation update," IEEE J. Oceanic Eng., vol. 0E-9, no. 3, pp. 179-188, 1984.

[11] R. Sadourny, A. Arakawa, and Y. Mintz, "Integration of the nondivergent barotropic vorticity equation with an icosahedral-hexagonal grid for the sphere," Mon. Wea. Rev., vol. 96, pp. 351-356, 1968.

[12] D. A. Randall, T. D. Ringler, and R. P. Heikes, "Climate modeling with spherical geodesic grids," Computing Sci. Engng., vol. 4, no. 5, pp. 32-41, Sep./Oct. 2002. 
[13] M. Brewer, L. Freitag-Diachin, P. Knupp, T. Leurent, and D. Melander, "The mesquite mesh quality improvement toolkit," in Proc. 12th Int. Meshing Roundtable, Santa Fe, NM, 2003, pp. 239-250.

[14] R. P. Heikes, "A Comparison of vertical coordinate systems for numerical modeling of the general circulation of the atmosphere," Ph.D. dissertation, Colo. State Univ., Fort Collins, CO, 2001.

[15] NOAA-NGDC, Global Relief CD-ROM.

[16] J. Hermance, "Electrical conductivity of the crust and mantle," in Global Earth Physics: A Handbook of Physical Constants. Washington, DC: AGU, 1995.

[17] P. Bannister, "The determination of representative ionospheric conductivity parameters for ELF propagation in the Earth-ionosphere waveguide," Radio Sci., vol. 20, no. 4, pp. 977-984, 1985.

[18] J. R. Wait, "On the theory of the slow tail portion of atmospheric wave forms," J. Geophys. Res., vol. 65, no. 7, pp. 1939-1946, 1960.

[19] M. A. Meju, "Geoelectromagnetic exploration for natural resources: models, case studies and challenges," Surveys Geophys., vol. 23, no. 2-3, pp. 133-205, 2002.

[20] E. P. Velikhov, A. A. Zhamaletdinov, A. N. Shevtsov, A. D. Tokarev, Y. M. Kononov, L. B. Pesin, G. M. Kadyshevich, M. I. Pertel, and A. V. Veshchev, "Deep electromagnetic studies with the use of powerful ELF radio installations," Isvestiya, Physics of the Solid Earth, vol. 34, no. 8, pp. 615-632, 1998.

[21] Y. B. Bashkuev and V. B. Khaptanov, "Deep radio impedance sounding of the crust using the electromagnetic field of a VLF radio installation," Izvestiya-Phys. of the Solid Earth, vol. 37, no. 2, pp. 157-165, 2001.

[22] U. S. Congress, Office of Technology Assessment, Oil production in the arctic national wildlife refuge: the technology and the Alaskan oil context OTA-E-394. Washington, DC, U.S., Government Printing Office, Feb. 1989.

[23] J. Garza, "Navy pulls plug on embattled ELF site," Milwaukee Journal Sentinel, Sep. 17, 2004 [Online]. Available: www.jsonline.com

[24] J. B. Lee, D. L. Dart, R. J. Turner, M. A. Downey, A. Maddever, G. Panjkovic, C. P. Foley, K. E. Leslie, R. Binks, C. Lewis, and W. Murray, "Airborne TEM surveying with a SQUID magnetometer sensor," Geophys., vol. 67, no. 2, pp. 468-477, Mar.-Apr. 2002.

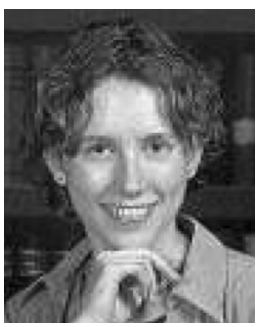

Jamesina J. Simpson (S'01) was born in Portsmouth, VA, on July 20, 1981. She is currently working toward the Ph.D. degree in electrical engineering at Northwestern University, Evanston, IL.

Since her freshman undergraduate year, she has been a Research Assistant in Prof. Taflove's Computational Electromagnetics Laboratory. Her current research interests include FDTD modeling of geophysically induced extremely low-frequency propagation about the entire Earth and FDTD modeling of novel ultrahigh-speed bandpass wireless interconnects for next-generation digital circuits.

Ms. Simpson is a member of Tau Beta Pi and Eta Kappa Nu. She received the National Science Foundation Graduate Research Fellowship, the 2004-2005 IEEE AP-S Graduate Research Award, the 2006 IEEE MTT-S Graduate Fellowship Award, and the Walter P. Murphy Fellowship for her graduate studies.

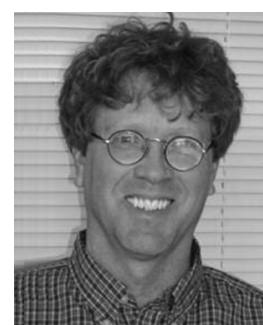

Ross P. Heikes received the B.S. degree in mathematics and the M.S. and Ph.D. degrees in atmospheric science from Colorado State University, Fort Collins, in 1989,1993 , and 2002 , respectively.

He is currently a Research Scientist in the Department of Atmospheric Science at Colorado State University. His research interests include numerical analysis, parallel computing and general circulation modeling.

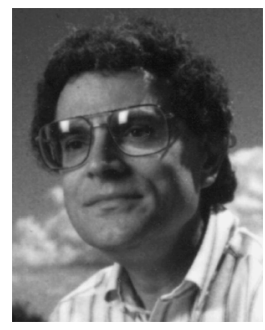

Allen Taflove (F'90) has pioneered basic theoretical approaches and engineering applications of finite-difference time-domain (FDTD) computational electrodynamics since 1972. He has been a Professor in the Department of Electrical Engineering and Computer Science at Northwestern University, Evanston, IL, since 1984. He coined the FDTD acronym in a 1980 IEEE paper, and in 1990 was the first person to be named a Fellow of the IEEE in the FDTD area. In total, he has authored or co-authored five books, 20 book chapters and articles, over 100 refereed journal papers and 300 conference papers, and 14 U.S. patents. These publications resulted in his being included on ISIHighlyCited.com, the Institute of Scientific Information's list of the most-cited researchers worldwide. $\mathrm{He}$ has been the adviser or co-advisor of $20 \mathrm{Ph}$.D. recipients and one postdoctoral fellow, five of whom (including four women) are now tenured or tenure-track university professors. His research interests span much of the electromagnetic spectrum. He and his students are modeling electrodynamic phenomena ranging from geophysically induced ultralow-frequency wave propagation about the entire Earth, to the light-scattering behavior of early-stage colon cancer cells. The principle that "Maxwell's equations work from dc to light" and especially for the benefit of human society is demonstrated in his laboratory every day.

Prof. Taflove is currently an elected member of Northwestern's General Faculty Committee. He is also the Faculty Advisor to the Undergraduate Design Competition, the Honors Program in Undergraduate Research, and the student chapters of Eta Kappa Nu and Tau Beta Pi. His efforts on behalf of students at all levels were recognized by Northwestern in 2000, when he was named a Charles Deering McCormick Professor of Teaching Excellence. 